\title{
The KNOMAD Methodology for Integration of Multi- Disciplinary Engineering Knowledge within Aerospace Production
}

\author{
Richard Curran ${ }^{1}$, Wim J.C. Verhagen ${ }^{2}$, Michel J.L. van Tooren ${ }^{3}$ \\ Delft University of Technology, Delft, 2629HS, The Netherlands
}

\begin{abstract}
The paper is associated with the integration of multi-disciplinary knowledge within a Knowledge Based Engineering (KBE)-enabled design framework. To support this integration effort, the KNOMAD methodology has been devised. KNOMAD stands for Knowledge Optimized Manufacture And Design and is a methodology for the analytical utilisation of multi-disciplinary engineering knowledge within design. The KNOMAD acronym can also be used to highlight KNOMAD's formalized process of: (K)nowledge capture; (N)ormalisation; (O)rganizations; (M)odelling; (A)nalysis; and (D)elivery. The main contribution of the paper is to highlight the development of the KNOMAD methodology and to substantiate its individual steps with sufficient detail to support the application of KNOMAD in practice.
\end{abstract}

\section{Nomenclature}

$\begin{array}{ll}D E E & =\text { Design and Engineering Engine } \\ D F M & =\text { Design for Manufacturing } \\ K B E & =\text { Knowledge Based Engineering } \\ K B S & =\text { Knowledge-Based Systems } \\ K M & =\text { Knowledge Management } \\ K N O M A D & =\text { Knowledge Optimized Manufacture and Design } \\ M D O & =\text { Multi-Disciplinary Optimization } \\ M P S & =\text { Manufacturing Process Simulation } \\ D E S & =\text { Discrete Event Simulation }\end{array}$

\section{Introduction}

$\mathrm{M}$

ANY projects within the aerospace sector are under high pressure to meet difficult production cost and rate objectives. Furthermore, environmental requirements are increasingly exerting their influence on product design, manufacturing and deployment. To achieve these objectives and comply with the myriad requirements of aircraft design, aerospace companies are increasingly turning towards the realization and implementation of an ability to carry out full optimization and integration for aerospace structures, including the rapid and automated assembly of structures and systems. This paves the way for adoption of established concurrent engineering paradigms within a software environment to achieve a more automated design process. Fully automated optimization and integration will require early and continuous use of Knowledge-Based Engineering (KBE) solutions that must be integrated over the various aircraft disciplines, including the disciplines that make up the design and manufacturing domains ${ }^{1}$. Over the last years, considerable progress has been made in establishing multi-disciplinary KBE solutions ${ }^{[1-3]}$. However, manufacturing engineering objectives and constraints in particular are still viable candidates for research and $\mathrm{KBE}$ implementation, as these manufacturing considerations are not or only marginally present in current $\mathrm{KBE}$

${ }^{1}$ Full Professor, Chair of Aerospace Transport \& Operations, Faculty of Aerospace Engineering, Kluyverweg 1, 2629 HS Delft, The Netherlands. R.Curran@tudelft.nl. Member AIAA.

${ }_{2}^{2}$ Ph.D. Student, Aerospace Transport \& Operations.

${ }^{3}$ Full Professor, Chair of Systems Engineering and Aircraft Design, Faculty of Aerospace Engineering. Member AIAA. 
applications ${ }^{2,3}$. Furthermore, existing KBE methodologies do not fully meet all requirements that must be met for a successful application of multi-disciplinary knowledge within early-stage design through KBE solutions.

This paper focuses on the integration of multi-disciplinary knowledge within a KBE-enabled design framework, which is formalized into a methodology known as KNOMAD. Knowledge Optimized Manufacture And Design (KNOMAD) is a methodology for the analytical utilization of multi-disciplinary knowledge within design and has been introduced and tentatively validated before ${ }^{4}$. The aim of this paper is to present an updated version of the KNOMAD methodology. This version goes beyond previous iterations by including detailed descriptions for the methodology steps. Furthermore, it improves upon existing KBE and MDO methodologies, as detailed in sections II and III.

First, research from relevant scientific disciplines will be presented. This includes a discussion of KnowledgeBased Systems, Knowledge Management and Knowledge-Based Engineering. After this theoretical context, the updated KNOMAD methodology is introduced in Section III. This updated methodology is subsequently detailed in Section IV. Relevant tools, techniques, examples and even methodologies are introduced to support the individual KNOMAD steps. Some conclusions are drawn with respect to the validity and applicability of the KNOMAD methodology. Finally, a perspective for future research is briefly discussed.

\section{Theoretical Context}

Increasing organizational and supply chain complexity, accelerating technical change and impending retirement or loss of company employees are often mentioned as drivers for companies to move towards the adoption of knowledge-based systems ${ }^{5,6}$. Knowledge-based systems use knowledge management methodologies and techniques to capture, store and use knowledge from various sources in order to be able to meet business objectives and requirements. This is reflected in the following definition of knowledge management": "Knowledge management is a systematic, organized, explicit and deliberate ongoing process of creating, disseminating, applying, renewing and updating intellectual and knowledge-based assets of the organization to achieve organizational objectives". In general, knowledge-based systems are set up to achieve one or more of the following objectives ${ }^{5}$ :

- Knowledge Capitalization: learning from the past by knowledge retention \& re-use.

- Project Accompaniment: learning from present activities by knowledge sharing and exchange.

- Innovation: moving towards future benefits by leveraging organizational knowledge assets.

- Cost reductions: achieving cost reductions through 'first-time right' adoption enabled by knowledge sharing.

Most knowledge-based systems using knowledge management methods and techniques are confronted by a number of challenges which relate to the type of knowledge that must be addressed. There are many knowledge type taxonomies proposed in literature ${ }^{7}$. However, Nonaka's explication ${ }^{8}$ of the epistemological dimension of organizational knowledge creation is one of the most used and applicable identifications of knowledge types. The epistemological dimension is split into explicit and tacit knowledge. Explicit knowledge refers to "articulated, codified knowledge that is communicated in symbolic or natural language form (e.g. reports, manuals)", while tacit knowledge refers to an individual's mental models (mental maps, beliefs, paradigms, viewpoints) and concrete know-how, crafts and skills that apply in a certain context ${ }^{8}$. Knowledge capture from explicit sources is complicated by the sheer amount of codified material that has to be analyzed and processed in order to retrieve the desired knowledge ${ }^{4}$. Furthermore, explicit sources often do not capture the tacit assumptions and decision trade-offs that underlie the captured, explicit knowledge. Knowledge capture from tacit sources faces other challenges ${ }^{9}$ : difficulties in explicating implicit knowledge, difficulties in retrieving truly useful knowledge and lack of engagement on the part of the tacit knowledge sources (e.g. experts).

Any knowledge-based application under development will require an investment of time and effort to retrieve the necessary explicit and tacit knowledge for meeting the application's objectives, while overcoming the challenges mentioned in this paragraph. This also holds for the specific case in which multi-disciplinary engineering knowledge has to be retrieved and implemented within Knowledge-Based Engineering (KBE) applications. Knowledge-Based Engineering ${ }^{10}$ is 'a technology based on the use of dedicated software tools that are able to capture and re-use 
product and process engineering knowledge. The main objective of KBE is reducing time and cost of product development by means of:

- Automation of repetitive, non-creative design tasks.

- Support of multi-disciplinary integration starting from the conceptual phase of the design process.'

The automation of repetitive, non-creative design tasks is often based on rule-based ${ }^{11}$ or parametric $^{1}$ approaches. In general, the user is freed from performing routine tasks, making more time available for truly creative design tasks and decisions.

A number of Knowledge-Based Engineering methodologies are available for use in applied projects. The main requirements for these methodologies are ${ }^{12}$ :

- Flexibility: methodology must be widely applicable to describe many different production system architectures, processes and product features.

- Extendibility: methodology must guarantee the possibility to rapidly extend the level of detail in the application.

- Scalability: methodology must be able to support the description of product, process and production systems at different levels of detail.

- Integration: interrelationships must be taken into the framework.

A number of KBE methodologies have been posited, for example the MOKA KBE Life-cycle ${ }^{13}$, where MOKA stands for Methodology and tools Oriented to Knowledge-based engineering Applications. This eight-step methodology (Identify, Justify, Capture, Formalize, Package, Distribute, Introduce, Use) is designed to take a project from inception towards industrialization and actual use ${ }^{13}$. When contrasting the MOKA methodology and other KBE methodologies (e.g. the KBE methodology for SME's ${ }^{14}$ ) to Colledani et al.'s list of requirements ${ }^{12}$, a number of major shortcomings of current KBE methodologies can be identified. First and foremost, current KBE methodologies are focused on establishing and maintaining KBE applications. Methodologies such as MOKA do not discuss the way in which the KBE applications are actually used in the design process. In particular, MOKA provides no perspective on integrating KBE applications within multi-disciplinary design optimization (MDO), an area of considerable interest for both research and practice ${ }^{2}$. To address this, TU Delft researchers have devised the Design and Engineering Engine (DEE) approach ${ }^{15-18}$ - see Figure 1.

The DEE consists of three major elements, as indicated in the figure. The first element is concerned with the design process, which includes multi-disciplinary optimization. After starting the design process by listing the requirements and making initial base-line calculations, an initiator containing product model parameter values is generated. This initiator feeds the parameter values to the second major element of the DEE, the Multi-Model Generator (MMG). The MMG is a modeling framework that uses the product model parameter values from the initiator in combination with formalized domain knowledge to generate product models. In effect, the MMG contains the fully formalized KBE applications that would result from applying the MOKA KBE Life-cycle. The MMG generates Report Files. These are fed to the third major element of the DEE: detailed analysis modules. These modules take the output from the MMG and calculate the design implications on a virtual per-discipline basis. Finally, the loop is closed by analyzing the Data files in a Converger \& Evaluator, which checks for mathematical validity (convergence) and requirements compliance (Evaluator) in a multi-disciplinary fashion. In effect, the current DEE iteration of the Converger \& Evaluator is a fully-fledged multi-disciplinary design optimizer. 


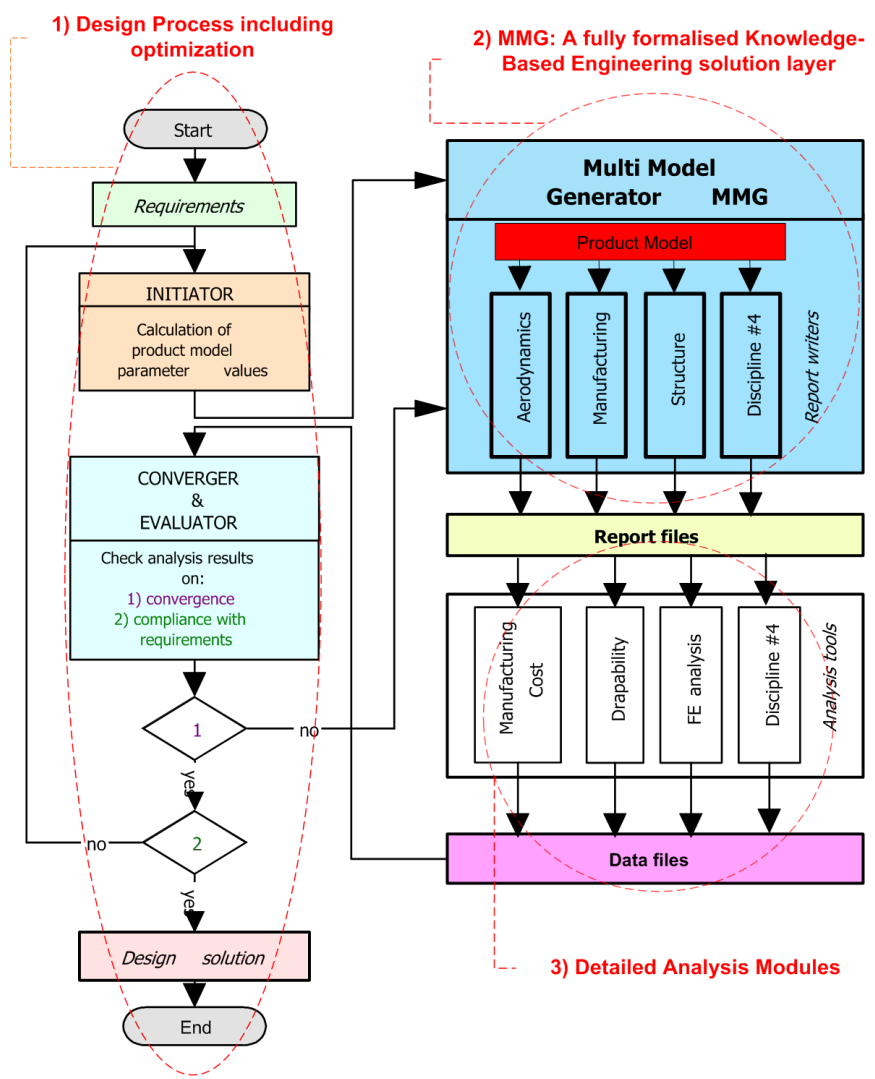

Figure 1: Design and Engineering Engine (DEE)

(Adapted from ref. 16)

To summarize, the DEE addresses KBE application in the form of the Multi-Model Generator, but extends beyond conventional KBE approaches such as MOKA by including detailed discipline analysis and subsequent multi-disciplinary optimization into its routine. However, the DEE does not include a methodological, formalized approach towards knowledge capture, knowledge formalization and knowledge delivery into business processes.

Existing KBE methodologies such as MOKA do have a methodological approach towards knowledge capture, formalization and delivery. However, this approach could be supported to a much higher degree. This assessment of course relates to the on-going identification of KBE methodology shortcomings: the second major shortcoming of existing KBE methodologies is the lack of substantiation for the recommended methodology steps. Relatively little tools and techniques are proposed. For instance, MOKA's 'Formalize' step is not accompanied by any tool or technique examples ${ }^{13}$; some basic advice about axiomization or use-case examples of successful programming implementations would enrich the methodology. Finally, another shortcoming is that the general scope and applicability of MOKA and other methodologies prevents them from offering more detailed and useful steps for particular disciplines.

To summarize, an improved methodology for the analytical utilization of multi-disciplinary engineering knowledge within design would have to:

- Include an approach for multi-disciplinary design (optimization).

- Include an approach for knowledge capture, formalization and delivery into business processes.

- Include significant substantiation of methodology steps; include examples, tools and techniques.

- Provide enough flexibility and scalability to enable discipline-specific application - e.g., the manufacturing discipline could be a use case 'instantiation' of the new methodology.

These requirements will be further addressed in Section III: The KNOMAD Methodology. 


\section{The KNOMAD Methodology}

The KNOMAD methodology has been devised ${ }^{4}$ to address the previously identified shortcomings of existing KBE methodologies and extend the scope of existing multi-disciplinary KBE-enabled optimization frameworks. An updated version of KNOMAD (with respect to ref. 4) that takes into account the full set of requirements summarized in section II is presented in this Section.

The principles underlying the DEE - in particular its advantages with respect to conventional KBE methodologies in terms of detailed discipline analysis and multi-disciplinary optimization - are used in combination with an approach towards knowledge capture, formalization and delivery to generate an improved methodology for the analytical utilization of multi-disciplinary engineering knowledge within design. This methodology has been named KNOMAD: Knowledge Optimized Manufacture and Design. It consists of the following steps (see also Figure 2):

- (K)nowledge capture: this first step comprises the identification of the scope, objective(s) and assumptions of the concerned project or use-case. Based on this, the required knowledge sources are identified. Subsequently, knowledge is captured, both from explicit sources (e.g. manuals, procedures) and tacit sources (e.g. domain experts, manufacturing engineers). Various knowledge elicitation techniques can be used to capture knowledge - see section IV for more detail. Finally, the captured knowledge has to be documented to support its use in the following steps.

- (N)ormalization: In the Normalization phase, the raw knowledge obtained in the knowledge capture phase is subjected to rigorous quality control and normalization. In this way, a sufficiently high quality level of knowledge content can be achieved, which enables seamless use of knowledge in subsequent organization, modeling and analysis. First, the set of raw knowledge is checked against applicable quality criteria to see whether it is fit for inclusion. Examples of quality criteria are knowledge reliability, and knowledge traceability (see section IV for more detail). Secondly, the captured knowledge is subjected to normalization, during which the knowledge is standardized for use in subsequent methodology steps.

- (O)rganisation: The organization of knowledge is an essential step towards knowledge utilization in modeling and analysis. Its purpose is to provide a knowledge structure that permits stakeholders from various disciplines to automatically access and retrieve the necessary knowledge for use in modeling and analysis. To achieve this, the construction of an ontology is an advisable method. An ontology is a 'explicit (formal) specifications of a conceptualization' ${ }^{19}$. In simpler terms, this indicates 'a way of thinking about a domain that is typically conceived and/or expressed as a set of concepts, their definitions and their inter-relationships' ${ }^{20}$. Construction of an ontology allows for formalization of knowledge through the use of rigorous definitions and axioms. The ontology forms the knowledge structure: the captured knowledge is instantiated within this structure. This knowledge (e.g. in the form of parameters) can be used to generate initiator values for the following KNOMAD step.

- (M)odeling: the next step in the KNOMAD methodology is the modeling of products and processes. Here, KNOMAD adopts the Multi-Model Generator (MMG) approach introduced in the DEE. As mentioned, the MMG is a modeling framework that uses product model parameter values in combination with formalized domain knowledge to generate product models. This approach can also be extended towards processes. Within the MMG definition, it is immediately evident that the ontology constructed in the previous step can be used to provide the product or process model parameters and any supporting formalized domain knowledge. The MMG produces models according to various disciplines (structures, aerodynamics, manufacturing, ...) and reports these in Report Files.

- (A)nalysis: analogous to the DEE approach, the Report Files containing the set of product and process models are used in detailed analysis modules. These modules calculate the design implications on a per-discipline basis. For instance, a manufacturing analysis module may calculate manufacturing costs, manufacturability estimates and manufacturing logistics. The Analysis step in KNOMAD can also comprises single- or multi-objective optimization with respect to the design objectives. As such, the KNOMAD Analysis step takes the DEE Converger \& Evaluator within its fold. In combination with the Modeling step, KNOMAD includes a full methodological approach towards multidisciplinary analysis and optimization.

- (D)elivery: Finally, the accepted optimized solution is delivered to the stakeholders and resource implications are evaluated. First, a design solution acceptance check is performed in which the design

5

American Institute of Aeronautics and Astronautics 
solution is compared with the requirements to determine the solution validity. After this, stakeholders need to receive, understand and act upon the design solution, as this has specific resource implications towards staff, consumables and production facilities. The resources can be managed in order to facilitate the realization of the optimized solution.

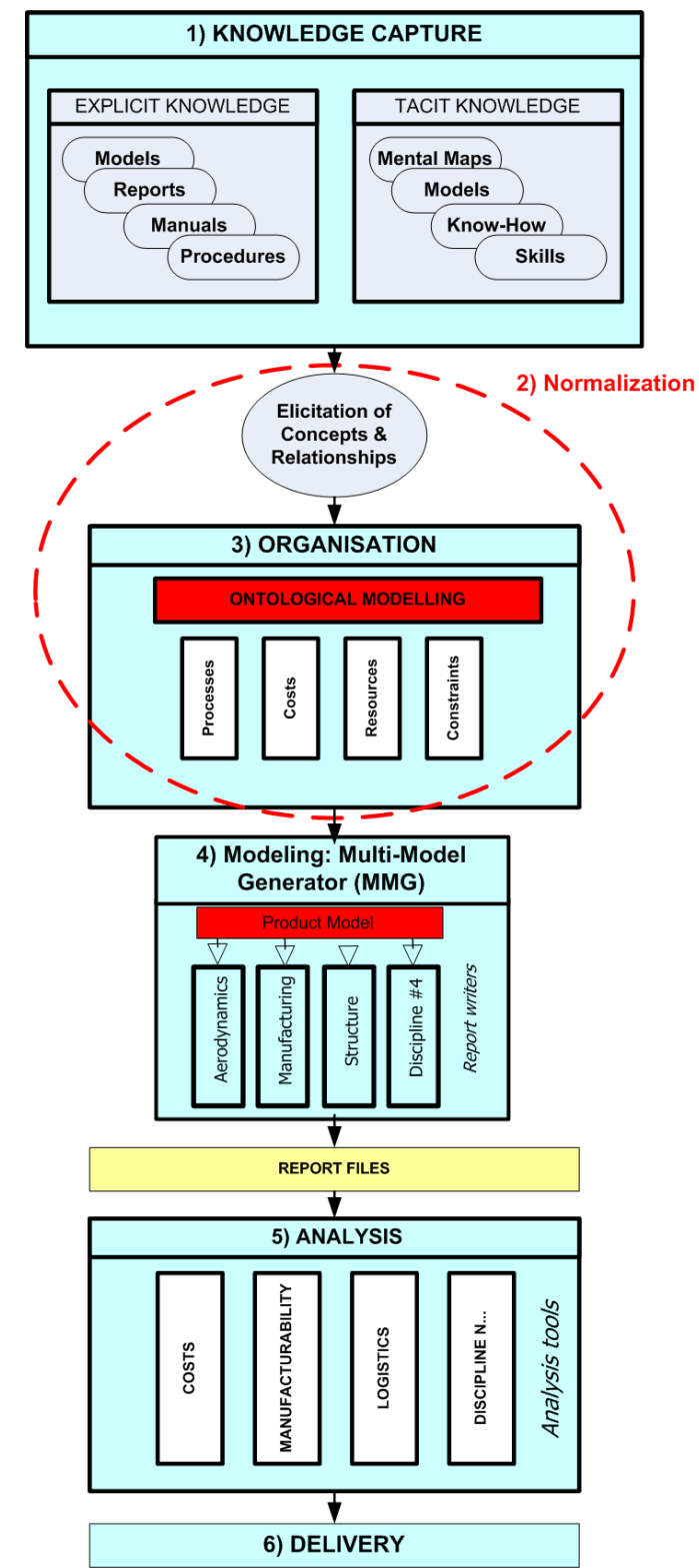

Figure 2: The KNOMAD Methodology

In short, KNOMAD addresses the requirements mentioned at the end of Section II. It improves upon existing methodologies by including multi-disciplinary (optimization) considerations, by including an approach for capturing, formalizing and delivering knowledge, and by providing enough flexibility and scalability to enable discipline-specific application. However, in its presented state KNOMAD retains one of the major disadvantages of existing KBE methodologies: lack of substantiation for the individual methodology steps. To remedy this, the individual KNOMAD steps are detailed in Section IV. 


\section{Detailing the KNOMAD Methodology}

In this Section, the individual KNOMAD methodology steps are substantiated. This is done through a discussion of each individual step, in which relevant tools, techniques, examples and even methodologies are presented.

\section{Step 1: (K)nowledge capture}

The knowledge capture step includes the identification of the scope, objective(s) and assumptions of the concerned project or use-case. Generally, an initial perspective on the scope, objectives and assumptions is available from the project customer, or from an initially defined mission need statement. To reach a final identification of scope, objectives and assumptions that is supported by all stakeholders, a combination of knowledge elicitation and systems engineering techniques can be used. For instance, brainstorming and mind-mapping can be employed to gain a fuller perspective on the involved aspects. Functional Breakdown Structures, Functional Flow Diagrams, organograms, and $\mathrm{N}^{2}$-charts are just a small selection of a wide range of system engineering techniques available to bring structure to the involved aspects. At the end of the process, scope, objective(s) and assumptions are agreed upon by all stakeholders. Based on this, the required knowledge sources are identified. This can be explicit and tacit knowledge sources. Subsequently, knowledge acquisition techniques can be applied to capture the knowledge. A wide range of knowledge acquisition techniques is available; a nice visual summary is provided by Epistemics ${ }^{21}$ (see Figure 3). For more detailed descriptions of the available techniques, see Ref. 21.

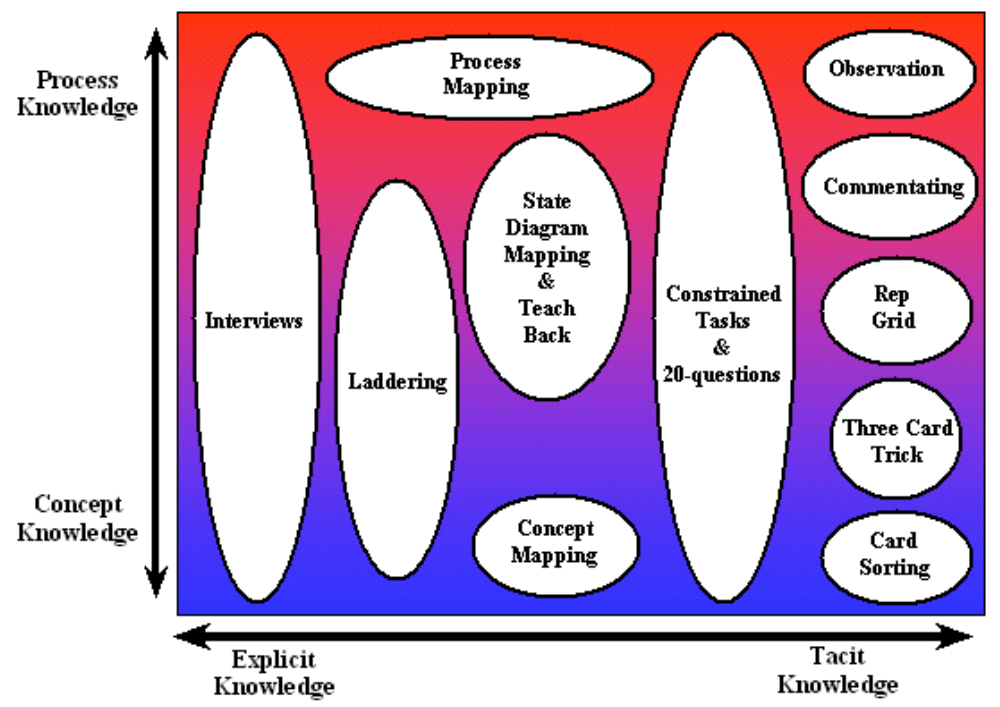

Figure 3: Knowledge Acquisition Techniques ${ }^{21}$

The final activity is to properly document the captured knowledge for use in the following KNOMAD steps. This means that knowledge has to be stored in a central repository from which it can be easily accessed. Options range from a collection of files stored in a project folder on a server to storage in a fully-fledged knowledge management environment.

\section{Step 2: (N)ormalization}

As mentioned in Section III, the objective of the normalization phase is to achieve a sufficiently high quality level of knowledge content to enable the seamless use of knowledge in subsequent organization, modeling and analysis. To achieve this, three distinct activities are performed. First, the knowledge is checked against applicable quality criteria. The following general quality criteria are recognized:

- Traceability: the knowledge must be traceable to its source and must be traceable over various iterations. This allows for capture of the associated design trade-offs, while giving insight into the overall knowledge life cycle. This benefits the design particularly in early-stage phases, when high 
numbers of iterations are performed: design decisions can be tracked to specific knowledge instances, allowing for faster learning and more insight in the overall design process.

- Ownership: the knowledge has to be tied to an owner. This owner takes responsibility for the accuracy and reliability of the knowledge. This is a prerequisite for efficient knowledge management, especially when tacit knowledge is involved. It can have significant benefits in later stages (organization, modeling, analysis) if necessary knowledge needs further explanation, as the owner can easily be contacted.

- Accuracy: is the captured knowledge accurate enough for subsequent use in modeling and analysis? The modeling and analysis of parameters puts requirements on accuracy, as significant variation or uncertainty in design parameters may prevent model and analysis resolution. For instance, modeling and analysis of the Friction Stir Welding (FSW) joining process requires definition of weld dimensions (length, width, and area), which must fall within specific limits ${ }^{15}$ for a successful weld given the constraints posed by the process and the available tooling, as well as the achievable tolerances.

- Reliability: how reliable is the captured knowledge? Particularly during early stage design, parameters have a tendency of being 'guesstimates', or they are founded on unchecked assumptions. Of course, designers vastly prefer confirmed parameters, but this is frequently not attainable during early design. If parameters are subject to high variation, this uncertainty should be taken into account during subsequent steps. In fact, the variation may be so high that the parameter in question is deemed too immature for further modeling and analysis. This approach benefits the overall process by limiting resources towards 'worthy' knowledge and by taking uncertainty into account, which leads to more reliable analysis estimates.

The second activity is normalization according to standards. The range of available standards to normalize against is considerable; the applicable standards for normalization depend on the context. Despite this, some examples of normalization through standardization may be given. A very easy example deals with the choice for SI units or Imperial units. This may seem trivial, but given today's heavily internationalized supply chains, a clear standard must be adopted and communicated to prevent problems. A brief inspection of areas in which standards must be set and/or adhered to gives

- Quality: for inclusion into further analysis, the knowledge must adhere to specific quality standards. Relevant quality criteria have already been mentioned. However, in terms of standardization, more formal requirements can be imposed on the knowledge. For instance, parameter distributions including acceptable variances may be imposed.

- Processes: numerous standards have been devised for processes and process quality. Well-known examples are the ISO process standards. These process standards can be applied to the captured knowledge to 'weed out' the knowledge that does not comply.

- Units: standardization in units includes the aforementioned choice for SI or Imperial units. Furthermore, unit standardization may include agreements about notation (e.g., the use of scientific notation, or even the use of the . or the, as a decimal operator).

- Regulations: dependent on the captured knowledge, it may be necessary to check for compliance with existing regulations. Examples within the aerospace community are the JAR and FAR regulations.

A final important activity within Normalization is to properly document the activities that are performed within this phase. The use of a dedicated knowledge management system may significantly ease the workload associated with documentation. Such a system may be supported by an organization of the knowledge in an appropriate structure as discussed in the next KNOMAD step.

\section{Step 3: (O)rganization}

As mentioned in Section III, the organization of knowledge is an essential step towards knowledge utilization in modeling and analysis. A knowledge structure must be provided that permits stakeholders from various disciplines to automatically access and retrieve the necessary knowledge for use in modeling and analysis. To do this, an ontology can be used. An ontology forms a formalized knowledge structure (through the use of concept definitions 
and interrelationships as expressed in axioms) that supports the instantiation of captured knowledge within the structure. As such, the KNOMAD Organization step must provide techniques, tools, and methodologies to support ontology construction. Fortunately, a significant amount of research has been performed on ontologies and their construction. For starters, there are a number of prominent ontology construction methodologies available. Examples include the methodologies by Uschold, Noy \& McGuinness, Uschold \& Gruninger and the METHONTOLOGY methodology $y^{20,22-24}$. As a visual example, the methodology by Uschold is represented in Figure 4.

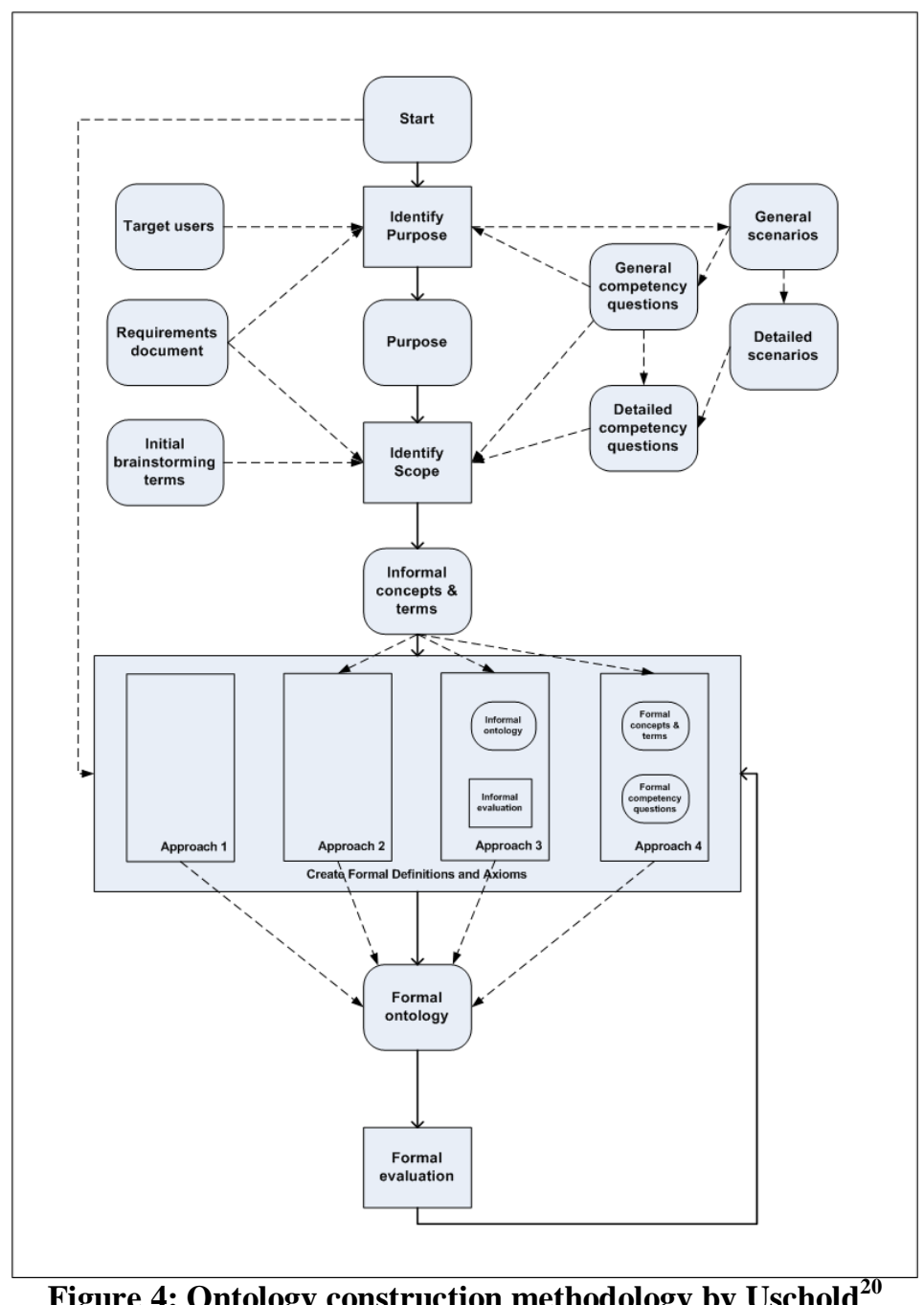

All of the aforementioned methodologies share common steps, though the exact representations may vary from methodology to methodology. The common steps are summarized by Pinto \& Martins ${ }^{25}$ :

- Specification: an identification of the purpose and scope of the ontology.

- Conceptualization: identification of the domain concepts and the relationships between concepts.

- Formalization: organizing the concepts into class hierarchies and subsequent construction of axioms to formally model the relationships between concepts.

- Implementation: codification of the class hierarchies and axioms into a formal knowledge representation language.

- Maintenance: updating and correcting the implemented ontology.

To further substantiate the Organization step, some examples are given with respect to the ontology construction methodology steps mentioned by Pinto \& Martins ${ }^{25}$. These examples are related to the aerospace composite 
manufacturing domain (scope), for a purpose beyond the interest of this paper. Keeping this specification in mind, Figure 5 shows a conceptualization of the aerospace composite manufacturing domain.

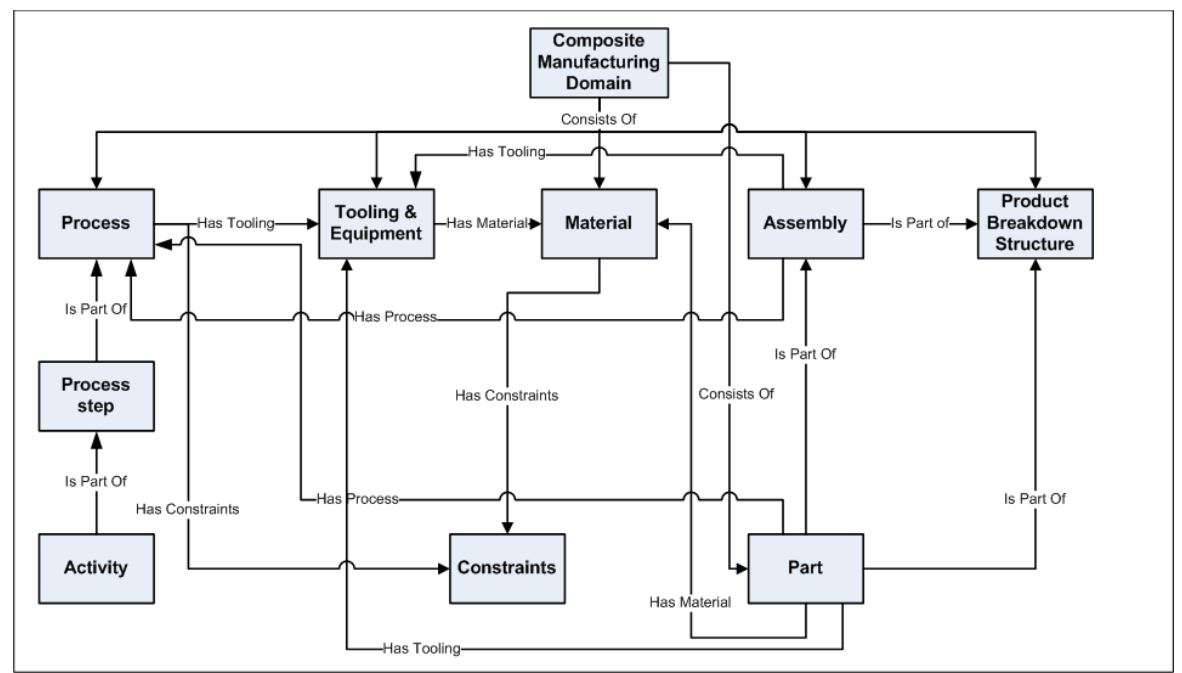

Figure 5: aerospace composite manufacturing domain conceptualization

In this figure, the relevant top-level domain concepts and relationships have been enumerated. The next step in a generic ontology construction methodology is to formalize the concepts into class hierarchies and construct axioms to accurately represent the concepts themselves and the relationships between concepts. This is followed by implementation within a formal knowledge representation language. These two steps are represented by Figure 6, which shows an implementation of the composite manufacturing domain ontology within Protégé-OWL. The toplevel domain concepts introduced in Figure 5 have been developed into classes with attendant class hierarchies, and are accompanied by classes and properties for resources such as costs (not all properties are shown, as this is prohibitive). For instance, the class 'Material' consists amongst others of the sub-class 'Composites', which in turn consists of the sub-classes 'Fiber Metal Laminates' and 'Fiber Reinforced Plastics'. The concept 'Composite' has been represented by an axiom which expresses that a material is regarded as a composite when it has both a fiber reinforcement and a matrix material. 


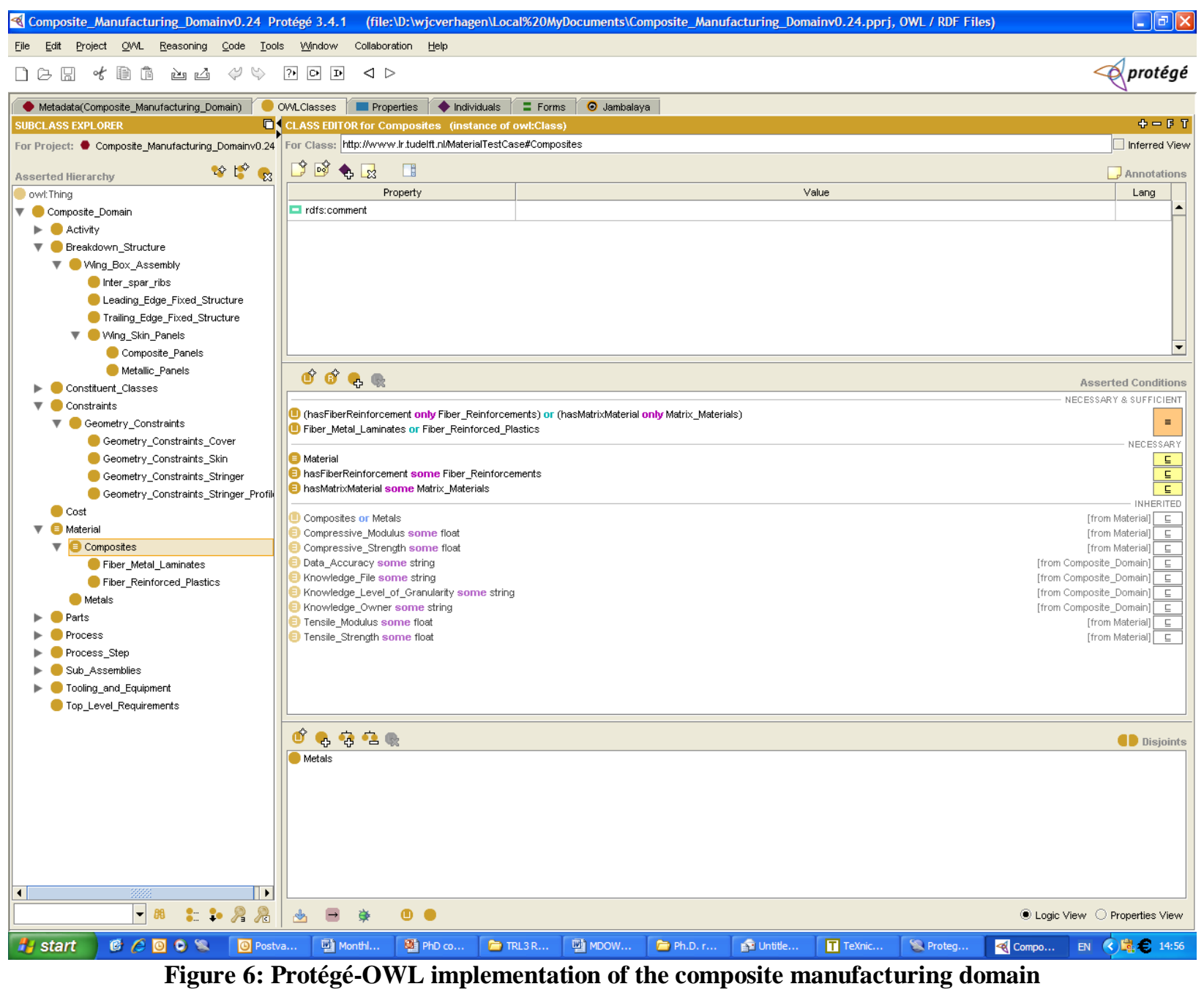

The final step of any ontology construction methodology relates to the maintenance of the ontology; its updating and correcting. A good example of this final step in practice is described in Ref. 26; the reader is referred to this paper for more detail.

\section{Step 4: (M)odeling}

The next step in the KNOMAD methodology is the modeling of products and processes. Here, KNOMAD adopts the Multi-Model Generator (MMG) approach introduced in the DEE. To support the MMG approach, the various disciplines for which a product or process should be modeled should be well understood ${ }^{2}$. Different actors from different disciplines in the design process have differing views on the same aircraft component; different aspects and details take precedence while designing an aircraft component. The specific requirements of a discipline can be used to generate discipline-specific models, which address the various needs from the involved actors. Also, having discipline-specific models is a prerequisite step on the path towards discipline-specific analysis. The MMG approach has been extensively employed within TU Delft PhD projects to model a range of products. As an example within the composite manufacturing domain, various discipline-specific parametric models for a composite wing moveable (Eaglet rudder) are represented in Figures 7-8. These figures are based on work by Van der Laan $(2008)^{2}$. 


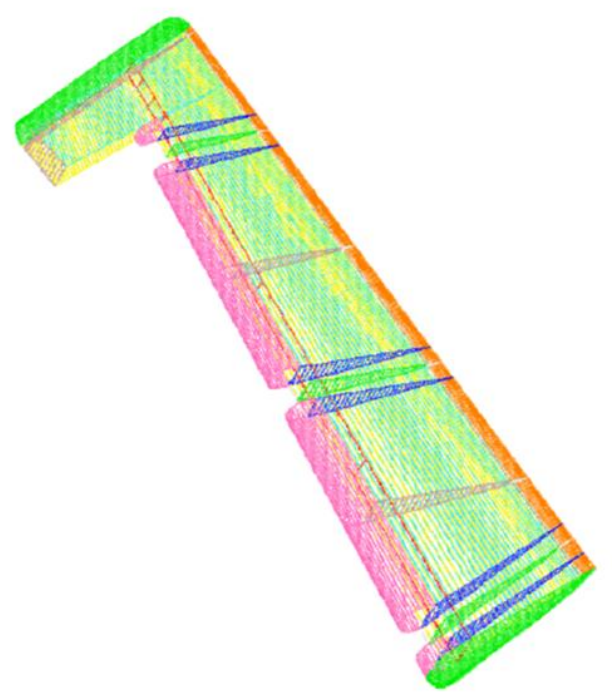

Figure 7: Structural View of multi-rib Eaglet rudder $^{2}$

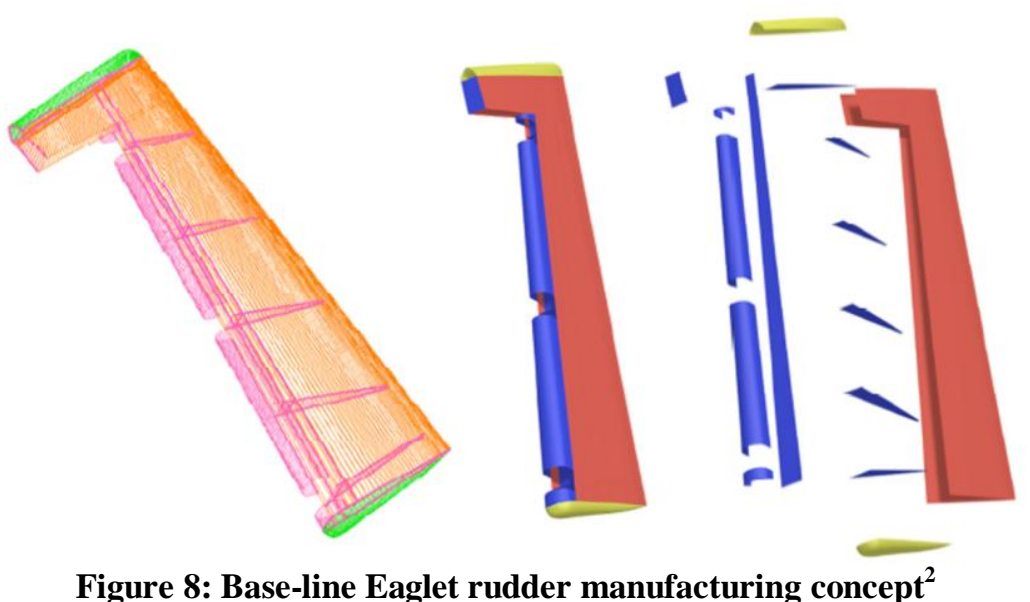

The multiple parametric models can be represented in Report Files, which are used to feed the detailed analysis modules.

\section{Step 5: (A)nalyis / (A)uthentication}

Analogous to the DEE approach, the Analysis step in KNOMAD employs Report Files containing the set of product and process models for use in detailed analysis modules. As mentioned before, these modules calculate the design implications on a per-discipline basis. As with the MMG, the requirements for analysis depend on the viewpoints of the involved stakeholders. Furthermore, the properties of the project (the scope, the objectives) and the product and process models themselves will steer analysis in certain directions. A cost analysis example is given with respect to the now-familiar composite manufacturing domain. Again, the Eaglet rudder is taken as a specific example. 

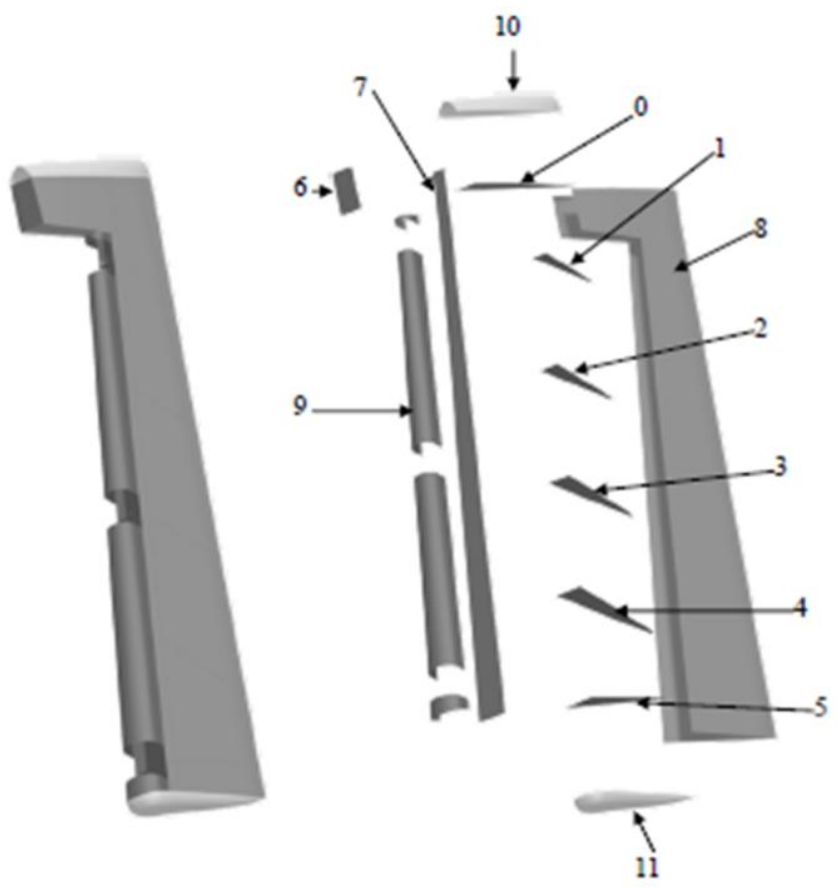

Figure 9: Base-line Eaglet rudder manufacturing view with part ID numbers ${ }^{2}$

Figure 9 again shows the base-line Eaglet rudder manufacturing view; the difference with respect to the previous representation is that part ID numbers have been assigned. The parts represented in this view have been subjected to cost analysis. The following cost analysis process has been employed (see Figure 10).

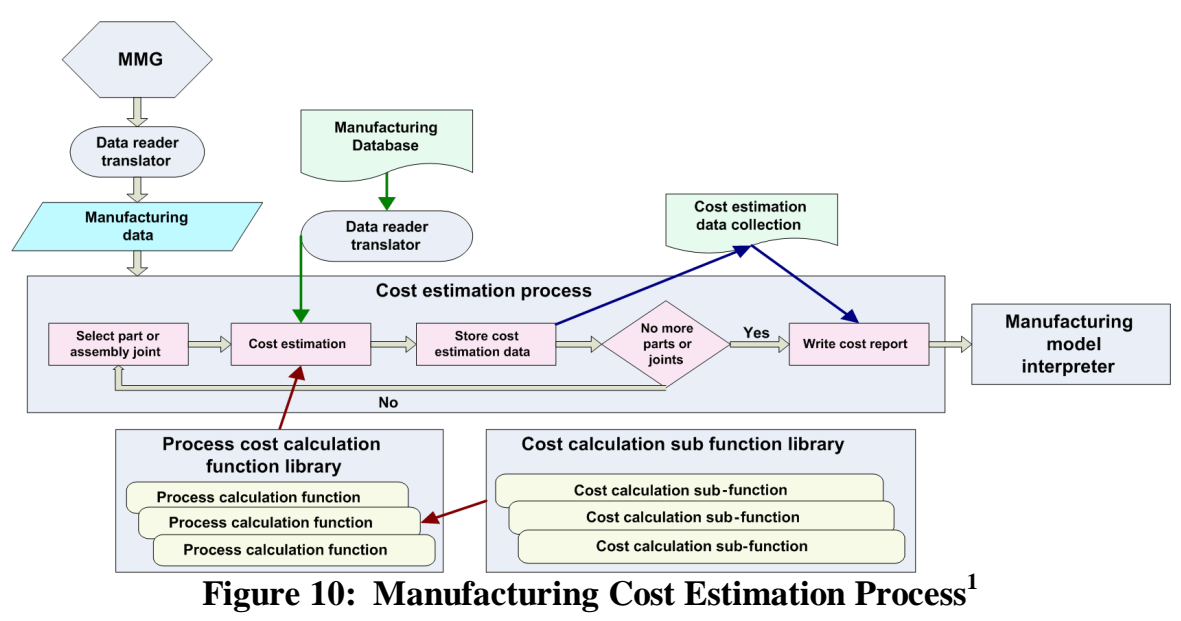

Various cost calculation functions have been applied to the various parts that make up the Eaglet rudder. The cost estimation process also took into account joining and assembly costs to accurately represent the total cost for production of one Eaglet rudder. The specifics of the cost estimation procedure are beyond the scope of this paper, but can be retrieved from Reference 2 . The calculated results are represented below in Table 1.

Table 1: Estimated cost of one baseline Eaglet rudder ${ }^{2}$

\begin{tabular}{|c|l|c|}
\hline Manufacturable part ID & \multicolumn{1}{|c|}{ Part Name } & Cost \\
\hline 0 & Upper Closure Rib & $€ 51$ \\
\hline 1 & Hinge Rib 1 & $€ 31$ \\
\hline 2 & Rib between Hinge 1 and 2 & $€ 34$ \\
\hline
\end{tabular}




\begin{tabular}{|c|l|c|}
\hline 3 & Hinge Rib 2 & $€ 39$ \\
\hline 4 & Rib between Hinge 2 and 3 & $€ 42$ \\
\hline 5 & Hinge 3 / Lower Closure Rib & $€ 44$ \\
\hline 6 & Horn Spar & $€ 33$ \\
\hline 7 & Main Spar & $€ 157$ \\
\hline 8 & Skin Panels & $€ 366$ \\
\hline 9 & Leading Edge (complete) & $€ 187$ \\
\hline 10 & Upper Endcap & $€ 144$ \\
\hline 11 & Lower Endcap & $€ 113$ \\
\hline & Manufacturable Parts Total & $€ 1,211$ \\
\hline & Assembly Total & $€ 813$ \\
\hline & Overall Total & $€ 2,024$ \\
\hline
\end{tabular}

Similar analyses can be run for different objectives, such as manufacturability (technical feasibility) estimates using commercially available Manufacturing Process Simulation (MPS) tools that can be used for analyses of drapeability, formability, and other process aspects. Also, time analysis is a prime candidate for analysis. Frequently, time analysis and cost analysis go hand in hand (aspects of time analysis have been used to estimate the assembly costs in the Eaglet rudder example).

\section{Step 6: (D)elivery}

The delivery step of KNOMAD starts with a check of the solution versus the requirements specified at the beginning of the design process. If this check is passed, the detailed analysis results can be delivered and resource implications can be evaluated. For example, process times and costs calculated in the Analysis step can be delivered directly ${ }^{27}$, or subjected to subsequent analysis such as Discrete Event Simulation (DES) to further explore the resource implications of a design. For instance, if DES is used, the logistical implications of a design on production space and lay-out can be explored in detail. Furthermore, a well set up ontological representation of the problem (as discussed in Organisation) will have included resource implications, either as a class in its own right with relationships to the product(s), process(es), tooling classes and so on, or as a property of the existing classes. This property has then 'accompanied' the instantiated objects during modeling and analysis in the subsequent KNOMAD phases, which will result in resource implications for each object.

\section{Conclusion}

This paper has presented an evolved version of the KNOMAD methodology. This methodology has been developed to improve upon existing KBE methodologies for the analytical utilization of multi-disciplinary engineering knowledge within design by addressing the following requirements:

1. Include an approach for multi-disciplinary design optimization.

2. Include an approach for knowledge capture, formalization and delivery into business processes.

3. Include significant substantiation of methodology steps; include examples, tools and techniques.

4. Provide enough flexibility and scalability to enable discipline-specific application.

The KNOMAD Knowledge Capture, Formalization and Organisation steps significantly improve upon existing methodologies by addressing requirement 2. The inclusion of the DEE principles (the use of the MMG and detailed analysis modules approaches) into the KNOMAD Modeling and Analysis steps addresses requirement 1 . This also provides the required flexibility and scalability to enable discipline-specific application. Finally, the methodology has been detailed in Section IV such that it offers a range of tools, techniques, (sub)-methodologies and examples for use towards KNOMAD application.

Further research into KNOMAD will have to focus on two areas. The first is methodology detailing: even though the first step has been taken in the paper, the detailing of the KNOMAD methodology can still be improved, particularly for the Delivery phase. Also, the Modeling and Analysis phases could benefit from a more structural approach (i.e. come with their own (sub-)methodologies). The second area for further research is validation: the KNOMAD methodology has to be validated through original use case studies. A number of case studies are currently under development.

14

American Institute of Aeronautics and Astronautics 


\section{Acknowledgements}

This work was conducted using the Protégé resource, which is supported by grant LM007885 from the United States National Library of Medicine.

\section{References}

${ }^{1}$ Van der Laan, A.H., \& Van Tooren, M.L.J., "Parametric modeling of moveables for structural analysis," Journal of Aircraft, $42(6), 2005$, pp. $1606-1614$.

${ }^{2}$ Van der Laan, A.H. Knowledge Based Engineering Support for aircraft component design, 2008, Delft, The Netherlands.

${ }^{3}$ Vermeulen, B., Knowledge Based Method for Solving Complexity in Design Problems, 2007, Delft, The Netherlands.

${ }^{4}$ Curran, R., Verhagen, W.J.C., Van der Laan, A.H., \& Van Tooren, M.L.J., "KBE and Manufacturing Constraints Management." In S. Chou, A. Trappey, J. Pokojski \& S. Smith (eds.), Global Perspective for Competitive Enterprise, Economy and Ecology: Proceedings of the 16th ISPE conference on Concurrent Engineering, 2009, Springer, London, pp. $783-791$.

${ }^{5}$ Ammar-Khodja, S., \& Bernard, A., “An overview on knowledge management”. In A. Bernard \& S. Tichkiewitch (eds.), Methods and Tools for Effective Knowledge Life-Cycle Management, 2008, Springer, Berlin, pp. 3-21.

${ }^{6}$ Hackbarth, G. "The impact of organizational memory on IT systems." In E. Hoadley \& I. Benbasat (eds.), Proceedings of the Fourth Americas Conference on Information Systems, 1998, pp. 588-590.

${ }^{7}$ Alavi, M., \& Leidner, D.E., "Review: Knowledge management and knowledge management systems: Conceptual foundations and research issues," MIS Quarterly, 25 (1), 2001, pp. 107-136.

${ }^{8}$ Nonaka, I., “A dynamic theory of organizational knowledge creation,” Organization Science, 5 (1), 1994, pp. 14-37.

${ }^{9}$ Kitamura, Y., Kashiwase, M., Fuse, M., \& Mizoguchi, R., "Deployment of an ontological framework of functional design framework," Advanced Engineering Informatics, 18 (2), 2004, pp. 115-127.

${ }^{10}$ La Rocca, G. KBE Techniques to support Aircraft Multi-Disciplinary Design and Optimization, Delft University of Technology, Delft, The Netherlands (unpublished).

${ }^{11}$ Mitchell, T., Buchanan, B., DeJong, G., Dietterich, T., Rosenbloom, P., \& Waibel, A., "Machine Learning". Annual Review of Computer Science, 4, 1990, pp. 417-433.

${ }^{12}$ Colledani, M., Terkaj, W., Tollio, T., \& Tomasella, M., "Development of a conceptual reference framework to manage manufacturing knowledge related to products, processes and production systems". In A. Bernard \& S. Tichkiewitch (eds.), Methods and Tools for Effective Knowledge Life-Cycle Management, 2008, Springer, Berlin, pp. 259-284.

${ }^{13}$ Oldham, K., Kneebone, S., Callot, M., Murton, A., \& Brimble, K., "MOKA - A methodology for developing KBE applications." Proceedings of the 8th European Conference on Product Data Technology, 1999, Stavanger, Norway, pp. 361366.

${ }^{14}$ Lovett, P.J., Ingram, A., Bancroft, C.N., "Knowledge-based engineering for SMEs -- a methodology", Journal of Materials Processing Technology, 107 (1-3), 2000, Pages 384-389.

${ }^{15}$ Van der Laan, A.H., Curran, R., Tooren, M.J.L. van., \& Ritchie, C., "Integration of friction stir welding into a multidisciplinary aerospace design framework," The Aeronautical Journal, 110 (1113), 2006, pp. 759-766.

${ }^{16}$ Lisandrin, P., Tooren, M.J.L. van., "Generic Volume Element Meshing for Optimization Applications", $9^{\text {th }}$ AIAA/ISSMO Symposium on Multidisciplinary Analysis and Optimisation, 2002, Atlanta, GA, USA, AIAA-2002-5647.

${ }^{17}$ La Rocca, G., Krakers, L.A., Tooren, M.J.L. van., "Development of an ICAD generative model for blended wing body aircraft design", $9^{\text {th }}$ AIAA/ISSMO Symposium on Multidisciplinary Analysis and Optimisation, 2002, Atlanta, GA, USA AIAA2002-5447. 
${ }^{18}$ Tooren, M.J.L. van, La Rocca, G., Krakers, L.A., Beukers, A., "Design and Technology in Aerospace: Parametric Modelling of Complex Structure Systems", The $14^{\text {th }}$ International Conference on Composite Materials (ICCM-14), San Diego, 2003. 220.

${ }^{19}$ Gruber, T., “A translation approach to portable ontology specifications,” Knowledge Acquisition, 5 (2), 1993, pp. 199 -

${ }^{20}$ Uschold, M., "Building Ontologies: Towards a Unified Approach", Proceedings of 16th Annual Conference of the British Computer Society Specialist Group on Expert Systems, 1996.

${ }^{21}$ Epistemics, Accessed 13-12-2009, URL: http://www.epistemics.co.uk/Notes/63-0-0.htm

${ }^{22}$ Uschold, M.,Gruninger, M., “Ontologies: Principles, Methods and Applications,” Knowledge Engineering Review, 11 (2), 1996, pp. $93-136$.

${ }^{23}$ Noy, N.F., McGuinness, D.L., “Ontology Development 101: A Guide to Creating Your First Ontology”, 2000, accessed 1012-2009, URL: http://www.ksl.stanford.edu/people/dlm/papers/ontology101/ontology101-noy-mcguinness.html

${ }^{24}$ Fernández López, M., Gómez-Pérez, A., Juristo, N., "METHONTOLOGY: From Ontological Art Towards Ontological Engineering”, Proceedings of the AAAI97 Spring Symposium, Stanford, USA, 1997

${ }^{25}$ Pinto, H.S., Martins, J.P., “Ontologies: How can they be Built?,” Knowledge and Information Systems, 6, 2004, pp. 441464 ,

${ }^{26}$ Rychtyckyj, N., "Measuring Long-Term Ontology Quality: A Case Study from the Automotive Industry", FLAIRS Conference, 2006, pp. 147-152.

${ }^{27}$ Curran, R, Raghunathan, S, and Price, M., "Review of Aerospace Engineering Cost Modelling: The Genetic Causal Approach,” Progress in Aerospace Sciences, Vol. 40, No. 8, 2004, pp. 487-534. 